\title{
The Safety of Tenofovir-Emtricitabine for HIV Pre- Exposure Prophylaxis (PrEP) in Individuals With Active Hepatitis B
}

\section{Citation}

Solomon, Marc M., Mauro Schechter, Albert Y. Liu, Vanessa M. McManhan, Juan V. Guanira, Robert J. Hance, Suwat Chariyalertsak, Kenneth H. Mayer, and Robert M. Grant. 2015. "The Safety of Tenofovir-Emtricitabine for HIV Pre-Exposure Prophylaxis (PrEP) in Individuals With Active Hepatitis B." Journal of Acquired Immune Deficiency Syndromes (1999) 71 (3): 281-286. doi:10.1097/QAl.0000000000000857. http://dx.doi.org/10.1097/QAl.0000000000000857.

\section{Published Version}

doi:10.1097/QAI.0000000000000857

\section{Permanent link}

http://nrs.harvard.edu/urn-3:HUL.InstRepos:26318770

\section{Terms of Use}

This article was downloaded from Harvard University's DASH repository, and is made available under the terms and conditions applicable to Other Posted Material, as set forth at http:// nrs.harvard.edu/urn-3:HUL.InstRepos:dash.current.terms-of-use\#LAA

\section{Share Your Story}

The Harvard community has made this article openly available.

Please share how this access benefits you. Submit a story.

Accessibility 


\title{
The Safety of Tenofovir-Emtricitabine for HIV Pre-Exposure Prophylaxis (PrEP) in Individuals With Active Hepatitis B
}

\author{
Marc M. Solomon, MD, MPH, *† Mauro Schechter, MD, PhD, $\neq$ Albert Y. Liu, MD, MPH, †\$ \\ Vanessa M. McManhan, MS,* Juan V. Guanira, MD, MPH,\| Robert J. Hance, AA,* \\ Suwat Chariyalertsak, MD, DrPH, 9 Kenneth H. Mayer, MD, \# and Robert M. Grant, MD, MPH, * for the \\ iPrEx Study Team
}

Background: Pre-exposure prophylaxis (PrEP) with daily oral emtricitabine and tenofovir disoproxil fumarate (FTC/TDF) prevents HIV infection. The safety and feasibility of HIV PrEP in the setting of hepatitis B virus (HBV) infection were evaluated.

\begin{abstract}
Methods: The Iniciativa Profilaxis Pre-Exposición study randomized 2499 HIV-negative men and transgender women who have sex with men to once-daily oral FTC/TDF versus placebo. Hepatitis serologies and transaminases were obtained at screening and at the time PrEP was discontinued. HBV DNA was assessed by polymerase chain reaction, and drug resistance was assessed by population sequencing. Vaccination was offered to individuals susceptible to HBV infection.
\end{abstract}

Results: Of the 2499 participants, $12(0.5 \%$; including 6 randomized to FTC/TDF) had chronic HBV infection. After stopping FTC/

Received for publication April 22, 2015; accepted September 1, 2015.

From the *Gladstone Institutes, San Francisco, CA; †University of California, San Francisco, San Francisco, CA; †़Projeto Praça Onze, Hospital Escola São Francisco de Assis, Universidade Federal do Rio de Janeiro, Rio de Janeiro, Brazil; §Bridge HIV, San Francisco Department of Public Health, San Francisco, CA; \|Investigaciones Medicas en Salud, Lima, Peru; IResearch Institute for Health Sciences and Department of Community Medicine, Faculty of Medicine, Chiang Mai University, Chiang Mai, Thailand; and \#Fenway Institute, Fenway Health, Beth Israel Deaconess Medical Center, and Harvard Medical School, Boston, MA.

Supported by DAIDS, NIAID, NIH (cooperative agreement UO1 AI64002 to R.M.G.), by the Bill \& Melinda Gates Foundation, and by T32AI007641 (Training Grant). Study drugs were donated by Gilead Sciences. Support for specimen handling came from a grant from DAIDS (RO1AI062333 to R.M.G.) and by the Gladstone Institutes. Some infrastructure support at the University of California, San Francisco, was provided by NIH (UL1 RR024131).

An oral abstract was presented at the International AIDS Society meeting, July 21, 2015, Vancouver, BC, Canada and was awarded the IAS/ANRS Lange-Van Tongeren Prize.

The authors have no conflicts of interest to disclose.

M.M.S. led the manuscript development. A.Y.L., M.M.S., M.S., and R.M.G. contributed to the data analysis and interpretation. M.S., A.Y.L., J.V.G., V. M.M., R.J.H., S.C., K.H.M., and R.M.G. contributed to site leadership, study oversight, study coordination, and manuscript development. R. M. G. reports personal fees from Siemens Healthcare, Merck, Clinical Care Options, and Medscape Education, outside the submitted work. K. M. has unrestricted research grants from Merck, Gilead and Bristol Myers Squibb. M. S. reports personal fees from Gilead Sciences.

Correspondence to: Marc M. Solomon, MD, MPH, 1650 Owens Street, 5th Floor, San Francisco, CA 94158 (e-mail: marc.solomon@ucsf.edu).

Copyright (C) 2015 Wolters Kluwer Health, Inc. All rights reserved. This is an open access article distributed under the terms of the Creative Commons Attribution-NonCommercial-NoDerivatives License 4.0 (CC BY-NC-ND), which permits downloading and sharing the work provided it is properly cited. The work cannot be changed in any way or used commercially.
TDF, 5 of the 6 participants in the active arm had liver function tests performed at follow-up. Liver function tests remained within normal limits at post-stop visits except for a grade 1 elevation in 1 participant at post-stop week 12 (alanine aminotransferase $=90$, aspartate aminotransferase $=61$ ). There was no evidence of hepatic flares. Polymerase chain reaction of stored samples showed that 2 participants in the active arm had evidence of acute HBV infection at enrollment. Both had evidence of grade 4 transaminase elevations with subsequent resolution. Overall, there was no evidence of TDF or FTC resistance among tested genotypes. Of 1633 eligible for vaccination, 1587 (97.2\%) received at least 1 vaccine; $1383(84.7 \%)$ completed the series.

Conclusions: PrEP can be safely provided to individuals with $\mathrm{HBV}$ infection if there is no evidence of cirrhosis or substantial transaminase elevation. HBV vaccination rates at screening were low globally, despite recommendations for its use, yet uptake and efficacy were high when offered.

Key Words: HIV, pre-exposure prophylaxis, hepatitis B, MSM, PrEP safety, transgender women

(J Acquir Immune Defic Syndr 2016;71:281-286)

\section{INTRODUCTION}

Antiretroviral pre-exposure prophylaxis (PrEP) with daily oral emtricitabine and tenofovir disoproxil fumarate (FTC/TDF) prevents acquisition of HIV infection in adults ${ }^{1-4}$ and is recommended by the Centers for Disease Control and Prevention (CDC) in the United States as part of a comprehensive package of preventative measures for individuals at substantial risk of HIV acquisition. ${ }^{5}$ FTC/TDF PrEP has an excellent safety profile and is well tolerated with subclinical effects on renal function ${ }^{6}$ and bone mineral density. ${ }^{7,8}$

FTC/TDF is also active against hepatitis B; however, concern has been raised that providing PrEP to individuals infected with hepatitis B could lead to hepatitis flares and injury, especially in the setting of suboptimal PrEP adherence or PrEP discontinuation. ${ }^{9}$ This concern stems from data on HIV-hepatitis B virus (HBV) coinfected individuals with chronic hepatitis B infections and cirrhosis who have high rates of hepatitis flares, sometimes leading to hepatic failure if they discontinue treatment for HIV with agents that are dually active against both HIV and HBV. ${ }^{10,11}$

The effect of PrEP use on hepatitis B infection is not well understood and most PrEP trials excluded participants with 
circulating HBV surface antigen at baseline., ${ }^{2,12}$ There are no reported cases of flares in HIV-uninfected persons with chronic hepatitis B infection who have discontinued FTC/TDF PrEP, although clinical experience with PrEP remains limited. Owing to the complexity of hepatitis B management, the CDC PrEP guidelines recommend that individuals positive for hepatitis $\mathrm{B}$ surface antigen be referred to a clinician who specializes in the treatment of hepatitis B before the initiation of PrEP. ${ }^{5}$

Furthermore, individuals susceptible to hepatitis B infection should be vaccinated, ${ }^{5}$ but recent data from the National Health and Wellness Survey suggest that only $26.3 \%$ of adults and $34.2 \%$ of men who have sex with men (MSM) in the US have ever received a hepatitis B vaccine. ${ }^{13}$ Although sparse, data on Latin American MSM cohorts also reveal low vaccination rates: only $7 \%$ in a cohort in Argentina reported ever receiving a hepatitis B vaccine. ${ }^{14}$ Even when HIV-infected individuals were found to be susceptible for hepatitis B, only $25 \%$ were vaccinated in a multicenter study of HIV-positive MSM in the US. ${ }^{15}$ PrEP delivery could offer an additional opportunity to evaluate hepatitis $B$ serostatus and increase vaccination rates.

We sough to provide information about hepatitis B global epidemiology, vaccine uptake and the safety of PrEP use among hepatitis B-infected men who have sex with men (MSM) and transgender women in the Iniciativa Profilaxis Pre-Exposición (iPrEx) study.

\section{METHODS}

The iPrEx study randomized 2499 MSM to evaluate the safety and efficacy of once-daily oral FTC/TDF PrEP for HIV prevention. ${ }^{2}$ Study visits were scheduled every 4 weeks after enrollment. $^{2}$ A secondary end point of the iPrEx study was the proportion of hepatic flares among subjects with active hepatitis B infection.

Hepatitis B surface antibody (HBsAb), hepatitis B core antibody ( $\mathrm{HBcAb}$ ), hepatitis B surface antigen (HBsAg), and hepatitis $\mathrm{C}$ antibody were obtained at screening of all participants. In addition, hepatitis serologies were repeated at the time PrEP was stopped if HBV infection status was ambiguous at baseline or if the study participants were susceptible at baseline. Participants at screening who were positive for $\mathrm{HBcAb}$ and negative for $\mathrm{HBsAb}$ were also tested for $\mathrm{HBcAb} \operatorname{IgM}(\mathrm{HBcAb} \operatorname{IgM})$ to distinguish acute from chronic infection. Individuals were excluded from the trial if the $\mathrm{HBcAb} \operatorname{IgM}$ was positive, if they had indications for $\mathrm{HBV}$ treatment based on local practice standards, or had elevations in liver function tests (LFTs), including alanine aminotransferase (ALT), aspartate aminotransferase (AST), or total bilirubin more than 2-fold the upper limit of normal (ULN) within 28 days of enrollment, or signs of hepatic cirrhosis. All participants with evidence of active hepatitis B infection were excluded from participation at sites in Brazil because of local concerns about safety and treatment monitoring requirements.

Hepatitis B e antigen ( $\mathrm{HBeAg}$ ) and hepatitis B e antibody $(\mathrm{HBe} A b)$ were tested for in enrolled participants with a positive HBsAg and when the activity of HBV remained ambiguous after initial screening (ie, when $\mathrm{HBcAb}$ was positive, $\mathrm{HBsAb}$ was negative, and $\mathrm{HBcAb}$ IgM was negative). Participants with a positive HBsAg were followed up with a medical history and
LFTs at weeks $4,8,12,16,20$, and 24 after stopping study drugs to assess for flares. Participants were also tested for elevated LFTs if they had signs or symptoms of hepatitis during the study and when deemed clinically appropriate.

For enrolled individuals with a positive HBsAg at any time during the trial, HBV DNA was measured via polymerase chain reaction. A HBV genotype was obtained for all participants with measurable HBV DNA.

Narratives were constructed for participants who were randomized to the FTC/TDF arm and were found to have acute hepatitis B infection.

Participants found to be HBV susceptible at screening were offered vaccination starting at their enrollment visit or at any time thereafter during study participation. When warranted by local medical practice standards, sites tested for $\mathrm{HBs} \mathrm{Ab}$ after vaccination and could provide an additional vaccine dose if $\mathrm{HBsAb}$ titers were insufficient. Flares were to be managed by specialists in internal medicine at the study site or referred to local specialists. Nucleoside and nucleotide analogue reverse transcriptase inhibitors were available for treatment of hepatitis B flares at study sites.

In a subset of participants, blood plasma was tested for the presence of FTC and tenofovir (TFV), and peripheral blood mononuclear cells were tested for FTC triphosphate and TFV diphosphate. ${ }^{16}$

\section{DEFINITIONS}

Active hepatitis B was defined as either acute hepatitis $\mathrm{B}$ or chronic hepatitis B infection.

Acute hepatitis B was defined by $\mathrm{HBcAb}, \mathrm{HBcAb}$ IgM and $\mathrm{HBsAg}$ positivity, and $\mathrm{HBsAb}$ negativity.

Chronic hepatitis B was defined by HBsAg and $\mathrm{HBcAb}$ positivity with $\mathrm{HBsAb}$ and $\mathrm{HBcAb}$ IgM negativity. Other hepatitis $\mathrm{B}$ designations are described in Table 1. A hepatic flare was defined as an increase in AST or ALT to more than 5 times ULN at any visit or $>2.5$ times ULN for 3 months, within 24 weeks of permanently discontinuing the study drug.

\section{RESULTS}

\section{Screening}

Four thousand nine hundred five individuals were screened for iPrEx. Baseline hepatitis B serologies were obtained from 4459 individuals (Table 2).

According to self-report, only $23.4 \%$ (1038/4431) had ever received a hepatitis $B$ vaccine of which less than half $45.7 \%$ (447/977) had received all 3 recommended vaccines. Vaccination rates varied widely by site (range: $20.6 \%-90.7 \%$ ), with US and Brazilian sites reporting the highest rates $(83 \%$ 91\%) and the Andean sites reporting the lowest (21\%-27\%).

\section{Enrollment \\ Chronic HBV Cases}

Among 2499 enrolled participants, 12 (0.48\%) (6 cases in each arm, mean age $=31$ [range: $20-54$ ] years) had chronic $\mathrm{HBV}$ infection, all with positive $\mathrm{HBsAg}$ and $\mathrm{HBcAb}$, and 
TABLE 1. Interpretation of the Hepatitis B Panel at Baseline

\begin{tabular}{lcc}
\hline Tests & Results & Interpretation and Management \\
\hline HBsAg & Negative & Susceptible. Offer vaccination \\
anti-HBc & Neries. If accepted, flag chart as \\
anti-HBs & "HBV vaccine series"; if \\
& vegative & the entire panel when stopping \\
& & study drug and assign HBV flare \\
& risk accordingly \\
HBsAg & Negative & Immune because of natural \\
anti-HBc & infection. Flag chart as "HBV \\
anti-HBs & Positive & Immune." No further testing \\
HBsAg & Positive & Immune because of hepatitis B \\
anti-HBc & Negative & vaccination. Flag chart as "HBV \\
anti-HBs & Negative & Immune." No further testing \\
HBsAg & Positive & Acutely infected, Flag chart as "At \\
anti-HBc & Positive & Risk for HBV Flare." Not \\
anti-HBs & Positive & eligible for enrollment
\end{tabular}

anti-HBc IgM Positive

HBsAg Positive

anti-HBc Positive

anti-HBs Negative

anti-HBc IgM Negative

HBsAg Negative

anti-HBc Positive

anti-HBs Negative

\begin{tabular}{|c|c|c|}
\hline $\begin{array}{l}\mathrm{HBsAg} \\
\text { anti-HBc }\end{array}$ & $\begin{array}{c}\text { Other result patterns } \\
\text { not listed above }\end{array}$ & Possible assay error \\
\hline
\end{tabular}

negative $\mathrm{HBs} \mathrm{Ab}$ and $\mathrm{HBcAb} \mathrm{IgM}$. Five were from the Andes (4 from Peru and 1 from Ecuador), 4 from Thailand, 1 from South Africa, and 2 from the US. All tested negative for hepatitis $\mathrm{C}$ antibodies.

\section{Chronic HBV Cases Randomized to FTC/TDF}

Of the $6 \mathrm{HBsAg}$-positive cases in the active arm, 5 were negative for $\mathrm{HBeAg}$ and positive for $\mathrm{HBeAb}$, and 1 was positive for $\mathrm{HBeAg}$ and negative for $\mathrm{HBeAb}$ (Figs. 1, 2A). The HBV DNA at baseline ranged from 0 to 8849 copies per milliliter in HBeAg-negative participants and was 336,000,000 copies per milliliter in the HBeAg-positive participants at baseline. Of the 5 individuals with $\mathrm{HBV}$ genotypes obtained, 3 had genotypes obtained after some exposure to FTC/TDF PrEP, and none had evidence of antiviral drug resistance. (One participant randomized to the placebo arm had the $80 \mathrm{P}$ mutation with possible resistance to lamivudine and telbivudine). After drug discontinuation, 5 of
TABLE 2. Hepatitis B Serologies Among Individuals Who Screened for iPrEx

\begin{tabular}{lc}
\hline & N (\%) \\
\hline Individuals screened for iPrEx & 4459 \\
Susceptible & $2828(63.4)$ \\
Immune because of natural infection & $794(17.8)$ \\
Immune because of hepatitis B vaccination & $529(11.9)$ \\
Infected with hepatitis B (acute and chronic) & $61(1.4)$ \\
$\quad$ Acutely infected & $22(0.5)$ \\
$\quad$ Chronically infected & $39(0.9)$ \\
Four interpretations possible & $246(5.5)$ \\
\hline
\end{tabular}

6 participants had LFTs performed at one or more post-stop follow-up visits (range: 6-7 visits). LFTs remained within normal limits at all post-stop visits for 5 of 6 participants, with no evidence of hepatic flares. In one participant randomized to FTC/TDF, grade 1 LFT elevations were seen at post-stop week 12 visit, with AST 61 (ULN 41) and ALT 90 (ULN 38); at the post-stop week 24 visit, AST was 37 and ALT was 60. These elevations did not meet protocol criteria for hepatic flares. ${ }^{17}$ Post-stop testing was not available in 1 participant who moved away. All 6 participants had evidence of detected drug at one or more visits. One participant with HBV viral load suppressed on FTC/TDF later developed a HBV viral rebound to 24,703 copies at a time when no drug was detected before permanent study drug discontinuation. The transaminases from the same date were ALT $=44$ and AST $=$ 51. Overall, while on randomized treatment, HBV viral loads ranged from 0 to 6205 at time points with detected drug and 0 to $>3.41 \times 10^{9}$ at time points with no detected drug.

\section{Narratives for 2 Acute HBV Cases Randomized to Receive FTC/TDF}

A 25-year-old man was randomized to FTC/TDF and dispensed study drug at enrollment (Fig. 2B). This participant was hepatitis B susceptible at screening (all negative for $\mathrm{HBsAg}, \mathrm{HBcAb}$, and HBsAb) with normal LFTs. At week 4, the participant had elevated LFTs $(\mathrm{AST}=205$, ALT $=669$ ), and serologies showed negative for $\mathrm{HBsAg}, \mathrm{HBsAb}, \mathrm{HBeAg}$; positive for $\mathrm{HBcAb}, \mathrm{HBcAb} \operatorname{IgM}, \mathrm{HBe} A b$ at that visit. LFTs normalized 2 weeks later, and by week 28 , he developed positive $\mathrm{HBs} \mathrm{Ab}$ and positive total $\mathrm{HBcAb}$ suggesting resolution of infection with protective immunity. Enrollment specimens were retrospectively tested and found to have an HBV viral load of 30,684, no evidence of resistance, HBsAg positive, $\mathrm{HBcAb}$ negative, HBsAb negative, and normal LFTs.

A 35-year-old participant was randomized to FTC/TDF and dispensed study drug on the day of enrollment. At screening, the participant had normal AST/ALT (35/35), with serologies consistent with chronic HBV infection: positive for $\mathrm{HBsAg}, \mathrm{HBcAb}$, and $\mathrm{HBeAg}$ and negative for $\mathrm{HBsAb}$, $\mathrm{HBcAb}$ IgM, and HBeAb. Testing at enrollment (15 days after his screening visit) indicated that his AST/ALT had increased to 214/304 (grade 3) before PrEP initiation. Eight days after enrollment, repeat LFTs at an interim visit indicated grade 4 elevations (ALT 1061, AST 1473), and PrEP was 

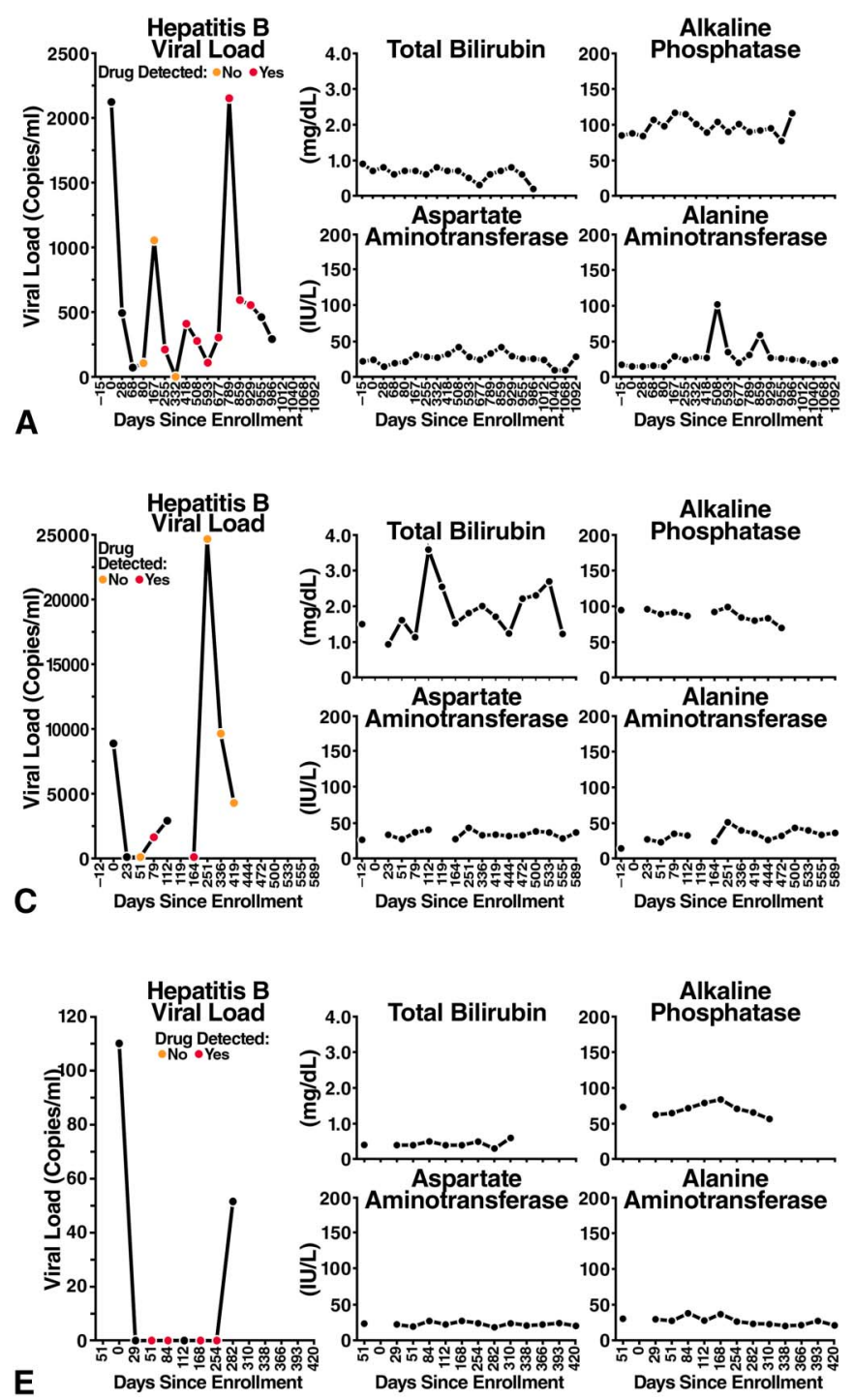

A-F, Hepatitis B V
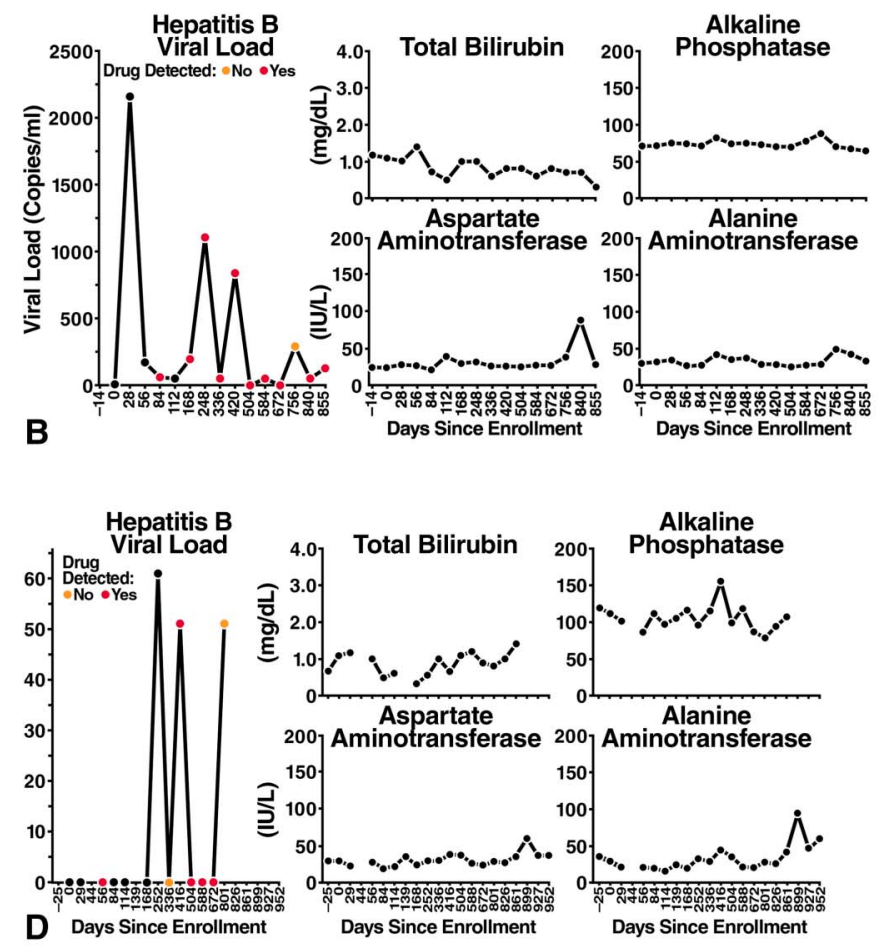

stopped. At that time, there was evidence of an acute HBV infection with interval appearance of detected $\mathrm{HBcAb} \operatorname{IgM}$ and continuing positive $\mathrm{HBsAg}, \mathrm{HBcAb}$, positive $\mathrm{HBeAg}$, and negative $\mathrm{HBsAb}$ and $\mathrm{HBeAb}$. Eleven weeks later, his AST/ ALT returned to normal, and he was restarted on study drug 3 weeks later. Retrospective analysis at enrollment revealed a HBV viral load $>3.41 \times 10^{9}$ copies that was undetectable by week 24 and remained undetectable for the duration of participant's time in the study. During visits at study weeks 28 and 72, HBV serology showed interval loss of HBsAg and $\mathrm{HBeAg}$ and interval appearance of $\mathrm{HBeAb}$, yet still no detectable $\mathrm{HBs} \mathrm{Ab}$. The patient self-terminated his participation in the study early at week 80 citing time constraints.

\section{Vaccine Uptake}

Of the 2499 enrolled participants, 1633 (65.4\%) were eligible for hepatitis B immunization. One thousand five hundred eighty-seven $(97.2 \%)$ participants received at least
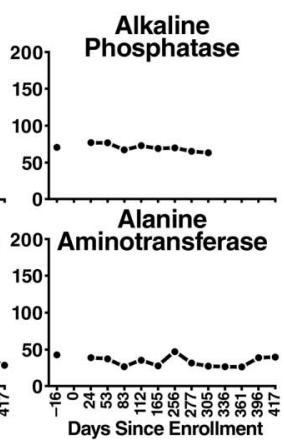

\section{$\mathbf{F}$}

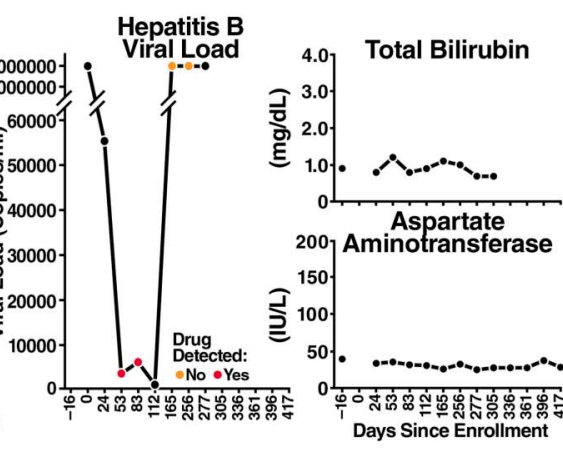

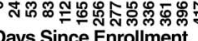

one hepatitis B immunization, and 1383 (84.7\% of total eligible) participants received the complete series of 3 injections.

\section{Vaccine Response}

Of the 1383 participants who received the complete hepatitis B vaccination series with 3 doses and postvaccination serologic testing, 86.9\% (1021/1175) developed immunity as measured by a positive HBsAb. 44.4\% (12/27) and $74.5 \%(38 / 51)$ of the individuals who received 1 or 2 doses, respectively, developed immunity. Two individuals who did not attain immunity were administered a fourth dose. Neither individual developed subsequent immunity.

\section{DISCUSSION}

In this large multinational randomized placebocontrolled clinical trial of oral FTC/TDF for HIV PrEP, hepatitis $\mathrm{B}$ exposure was highly prevalent $(>25 \%$ of 


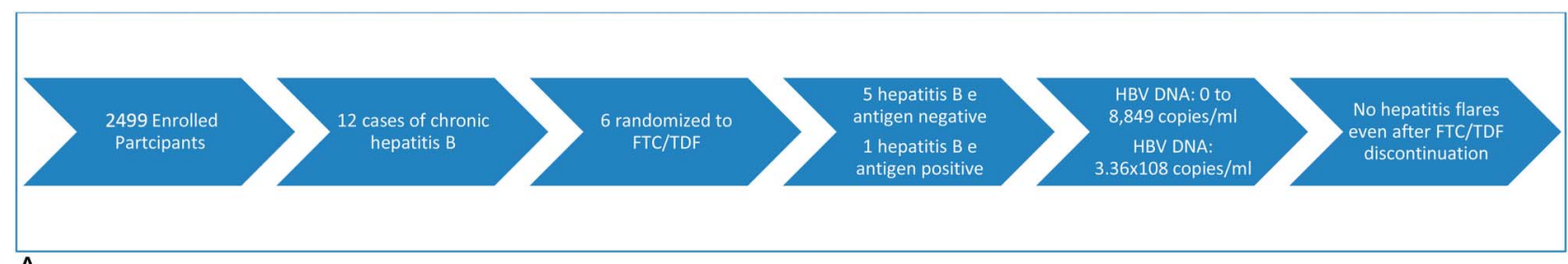

A

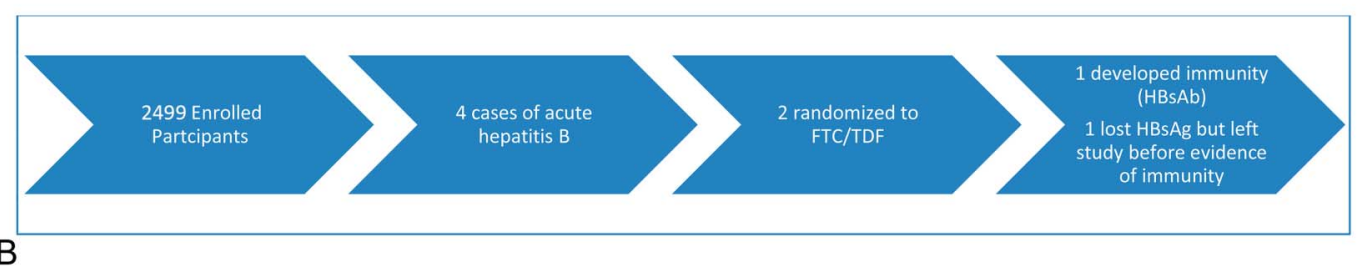

FIGURE 2. A, Cases of chronic hepatitis B infection randomized to FTC/TDF. B, Cases of acute hepatitis B infection randomized to FTC/TDF.

participants screened with evidence of prior or active infection). Although over $60 \%$ of the participants were HBV susceptible at screening, fewer than $25 \%$ had reported ever receiving an $\mathrm{HBV}$ vaccine. Among those who had received a vaccine, fewer than half had received all 3 doses recommended by the $\mathrm{CDC}^{18}$; this deficiency varied widely among the sites and was especially high in the Andes.

In contrast to low vaccination rates before the trial, vaccine uptake during the trial was excellent with over $97 \%$ of eligible participants receiving at least 1 dose and $85 \%$ receiving the complete series; the vaccine response rate (as reflected by $\mathrm{HBsAb}$ seroconversion) for those completing the series was $87 \%$, consistent with the CDC data regarding vaccine immunogenicity. ${ }^{19}$ The acute $\mathrm{HBV}$ infections that occurred during the study highlight how prevention through vaccination is imperative, and the low rates of global vaccination provide a clear opportunity for disease prevention. Engaging adult MSM and transgender women in PrEP programs may offer a unique opportunity to increase vaccination rates. Interestingly, there is also some evidence from a recent retrospective study that use of hepatitis B-active antiretroviral therapy for treatment of HIV (lamivudine, emtricitabine, and TFV) led to a lower incidence of hepatitis $B$ acquisition than the use of antiretrovirals without hepatitis B activity. ${ }^{20}$ This effect was especially apparent when TFV was used, which provides another protective benefit of FTC/TDF use and is consistent with our data, showing that no acute HBV infections occurred after 4 weeks on FTC/TDF; both cases of acute $\mathrm{HBV}$ infection that occurred in the placebo arm occurred later. These findings suggest that FTC/TDF might serve to prevent hepatitis B-susceptible people from acquiring hepatitis B before completion of the vaccine series.

Among the 12 participants enrolled in the study with chronic hepatitis $\mathrm{B}$, almost all of those tested had no evidence of antiviral drug resistance (8/9). One isolate from a participant randomized to the placebo arm showed possible resistance to lamivudine and telbivudine. No participant had virus associated with resistance mutations against TFV, and no participants experienced a flare either during the trial or after drug discontinuation, although one participant whose LFTs were normal while on study drug developed a low-grade, asymptomatic, and self-limited elevation of the transaminases after PrEP discontinuation. The majority with chronic hepatitis B randomized to FTC/TDF (5 of 6) had visits when drug was detected followed by visits when no drug was detected, indicating inconsistent adherence, and yet, none experienced a hepatitis B flare. It is possible that flares were absent because individuals with evidence of liver dysfunction were excluded. The risk of flares with FTC discontinuation seems to be limited to people with advanced liver disease. ${ }^{21}$

Interestingly, TFV use in chronic hepatitis B in the setting of cirrhosis has led to regression of fibrosis. ${ }^{22}$ Regarding the 2 cases of acute HBV infection within the FTC/TDF arm, 1 resolved with development of immunity and the other resolved clinically with loss of circulating antigen but remained $\mathrm{HBsAb}$ negative. It is not known whether this person may have a recurrence of HBV antigenemia after stopping PrEP.

The principal limitation of this study is the small number of enrolled participants with active HBV infection, which limits the ability to provide definitive conclusions regarding the safety of PrEP use in the context of HBV infection in MSM/TGW or conclusive guidance related to best practices. Our results are reassuring, however, in that none of the participants in iPrEx with chronic HBV infection developed a flare during intermittent use of PrEP and even after drug discontinuation.

We agree with the CDC that screening for HBsAg should occur before PrEP initiation; while most providers prescribing PrEP are compliant with most PrEP guidelines, only approximately $60 \%$ of providers are screening for HBV infection. ${ }^{23}$ Obtaining HBsAg alone followed by HBV DNA (when available) for individuals with a positive HBsAg may be the only required testing before initiating PrEP, especially in resource-limited settings in which obtaining additional serologies may be more expensive than vaccination.

We did not find that testing for $\mathrm{HBsAb}, \mathrm{HBcAb}$, $\mathrm{HBcAb} \mathrm{IgM}$, $\mathrm{HBeAb}$, or $\mathrm{HBeAg}$ added value for the purposes of assessing PrEP eligibility. Importantly, persons with isolated $\mathrm{HBcAb}$ (and negative $\mathrm{HBsAg}$ and $\mathrm{HBsAb}$ ) were all HBV DNA negative with normal LFTs, indicating that this common pattern of serologies was consistent immunity from 
prior infection rather than active infection with a $\mathrm{HBV}$ mutant. Individuals with $\mathrm{HBV}$ infection who are interested in PrEP can be safely started on FTC/TDF, which will also provide excellent activity against $\mathrm{HBV}^{24}$; consistent adherence may be required to prevent development of resistance.

This experience, combined with prior evidence showing no hepatitis flares among 23 women during and after oral TDF PrEP ${ }^{12}$ demonstrates that PrEP can often be safely started and stopped in persons with HBV infection who do not have cirrhosis or transaminase elevations at PrEP initiation. As information is limited and treatment for $\mathrm{HBV}$ infection is complex, referral to a specialist is appropriate when available, especially for PrEP discontinuation. At that time, a separate decision regarding continuing FTC/TDF or switching management strategies for $\mathrm{HBV}$ infection must be reached. ${ }^{5}$ Such a decision would be based on further testing to evaluate cirrhosis to determine the need for ongoing hepatitis B treatment. ${ }^{25,26}$

Individuals infected with HBV should have monitoring of LFTs (and possibly HBV viral load where available) every 6-12 months while on PrEP to ensure that the emergence of $\mathrm{HBV}$ resistance to $\mathrm{FTC} / \mathrm{TDF}$ has not occurred, a theoretical concern with suboptimal adherence. ${ }^{5}$ Although TDF presents a high barrier to developing HBV drug resistance, especially when given as combination therapy, ${ }^{27}$ inconsistent adherence due to intermittent dosing may lead to drug resistance, ${ }^{28}$ yet no cases have been reported. We did not observe any TDF resistance during the $\mathrm{iPrEx}$ study during which adherence to the daily regimen occurred in a minority of participants. ${ }^{2}$

\section{ACKNOWLEDGMENTS}

The authors thank the study participants for their dedication to HIV prevention; the community advisory boards; the members of the DAIDS multinational independent data and safety monitoring board; Megha Mehrotra for data quality assurance; John Carroll for assistance with graphics. Brian Postle for database management and data transfer. Vera Holzmeyer and Catherine Brennan at Abbott Laboratories for $H B V$ viral load and resistance testing. The following are additional members of the iPrEx Study Team who contributed to this work: Linda-Gail Bekker, Susan Buchbinder, Martin Casapia, Esper Kallas, Javier Lama, Orlando Montoya, and Valdiléa Veloso.

\section{REFERENCES}

1. Baeten JM, Donnell D, Ndase P, et al. Antiretroviral prophylaxis for HIV prevention in heterosexual men and women. $N$ Engl J Med. 2012;367: 399-410.

2. Grant RM, Lama JR, Anderson PL, et al. Preexposure chemoprophylaxis for HIV prevention in men who have sex with men. $N$ Engl J Med. 2010; 363:2587-2599.

3. Thigpen MC, Kebaabetswe PM, Paxton LA, et al. Antiretroviral preexposure prophylaxis for heterosexual HIV transmission in Botswana. N Engl J Med. 2012;367:423-434.

4. Choopanya K, Martin M, Suntharasamai P, et al. Antiretroviral prophylaxis for HIV infection in injecting drug users in Bangkok, Thailand (the Bangkok Tenofovir Study): a randomised, double-blind, placebocontrolled phase 3 trial. Lancet. 2013;381:2083-2090.

5. CDC. Preexposure Prophylaxis for the Prevention of HIV Infection in the United States-2014 Clinical Practice Guideline. Atlanta, GA; Centers for Disease and Control: 2014.
6. Solomon MM, Lama JR, Glidden DV, et al. Changes in renal function associated with oral emtricitabine/tenofovir disoproxil fumarate use for HIV pre-exposure prophylaxis. AIDS. 2014;28:851-859.

7. Liu AY, Vittinghoff E, Sellmeyer DE, et al. Bone mineral density in HIV-negative men participating in a tenofovir pre-exposure prophylaxis randomized clinical trial in San Francisco. PLoS One. 2011;6:e23688.

8. Kasonde M, Niska RW, Rose C, et al. Bone mineral density changes among HIV-uninfected young adults in a randomised trial of pre-exposure prophylaxis with tenofovir-emtricitabine or placebo in Botswana. PLoS One. 2014;9:e90111.

9. Levy V, Grant RM. Antiretroviral therapy for hepatitis B virus-HIVcoinfected patients: promises and pitfalls. Clin Infect Dis. 2006;43:904-910.

10. Bessesen M, Ives D, Condreay L, et al. Chronic active hepatitis B exacerbations in human immunodeficiency virus-infected patients following development of resistance to or withdrawal of lamivudine. Clin Infect Dis. 1999;28:1032-1035.

11. Mondou E, Sorbel J, Anderson J, et al. Posttreatment exacerbation of hepatitis B virus (HBV) infection in long-term HBV trials of emtricitabine. Clin Infect Dis. 2005;41:e45-e47.

12. Peterson L, Taylor D, Roddy R, et al. Tenofovir disoproxil fumarate for prevention of HIV infection in women: a phase 2, double-blind, randomized, placebo-controlled trial. PLoS Clin Trials. 2007;2:e27.

13. Annunziata K, Rak A, Del Buono H, et al. Vaccination rates among the general adult population and high-risk groups in the United States. PLoS One. 2012;7:e50553.

14. Segura M, Bautista CT, Marone R, et al. HIV/STI co-infections, syphilis incidence, and hepatitis B vaccination: the Buenos Aires cohort of men who have sex with men. AIDS Care. 2010;22:1459-1465.

15. Hoover KW, Butler M, Workowski KA, et al. Low rates of hepatitis screening and vaccination of HIV-infected MSM in HIV clinics. Sex Transm Dis. 2012;39:349-353.

16. Anderson PL, Glidden DV, Liu A, et al. Emtricitabine-tenofovir concentrations and pre-exposure prophylaxis efficacy in men who have sex with men. Sci Transl Med. 2012;4:151ra125.

17. Avihingsanon A, Matthews GV, Lewin SR, et al. Assessment of HBV flare in a randomized clinical trial in HIV/HBV coinfected subjects initiating HBV-active antiretroviral therapy in Thailand. AIDS Res Ther. 2012;9:6.

18. Mast EE, Margolis HS, Fiore AE, et al. A comprehensive immunization strategy to eliminate transmission of hepatitis B virus infection in the United States: recommendations of the Advisory Committee on Immunization Practices (ACIP) part 1: immunization of infants, children, and adolescents. MMWR Recomm Rep. 2005;54:1-31.

19. Centers for Disease Control and Prevention. Epidemiology and Prevention of Vaccine-Preventable Diseases. Hamborsky J, Kroger A, Wolfe S, eds. 12th ed. Washington D.C. Public Health Foundation, 2012.

20. Heuft MM, Houba SM, van den Berk GE, et al. Protective effect of hepatitis B virus-active antiretroviral therapy against primary hepatitis B virus infection. AIDS. 2014;28:999-1005.

21. Thio CL, Sulkowski MS, Thomas DL. Treatment of chronic hepatitis B in HIV-infected persons: thinking outside the black box. Clin Infect Dis. 2005;41:1035-1040.

22. Marcellin P, Gane E, Buti M, et al. Regression of cirrhosis during treatment with tenofovir disoproxil fumarate for chronic hepatitis B: a 5year open-label follow-up study. Lancet. 2013;381:468-475.

23. Tellalian D, Maznavi K, Bredeek UF, et al. Pre-exposure prophylaxis (PrEP) for HIV infection: results of a survey of HIV healthcare providers evaluating their knowledge, attitudes, and prescribing practices. AIDS Patient Care STDS. 2013;27:553-559.

24. Marcellin P, Heathcote EJ, Buti M, et al. Tenofovir disoproxil fumarate versus adefovir dipivoxil for chronic hepatitis B. $N$ Engl J Med. 2008; 359:2442-2455.

25. Lok AS, McMahon BJ. Chronic hepatitis B: update 2009. Hepatology. 2009;50:661-662.

26. Morgan M, Keeffe EB. Diagnosis and treatment of chronic hepatitis B: 2009 update. Minerva Gastroenterol Dietol. 2009;55:5-22.

27. Hongthanakorn C, Chotiyaputta W, Oberhelman K, et al. Virological breakthrough and resistance in patients with chronic hepatitis $\mathrm{B}$ receiving nucleos(t)ide analogues in clinical practice. Hepatology. 2011;53:1854-1863.

28. Sheldon J, Camino N, Rodes B, et al. Selection of hepatitis B virus polymerase mutations in HIV-coinfected patients treated with tenofovir. Antivir Ther. 2005;10:727-734. 